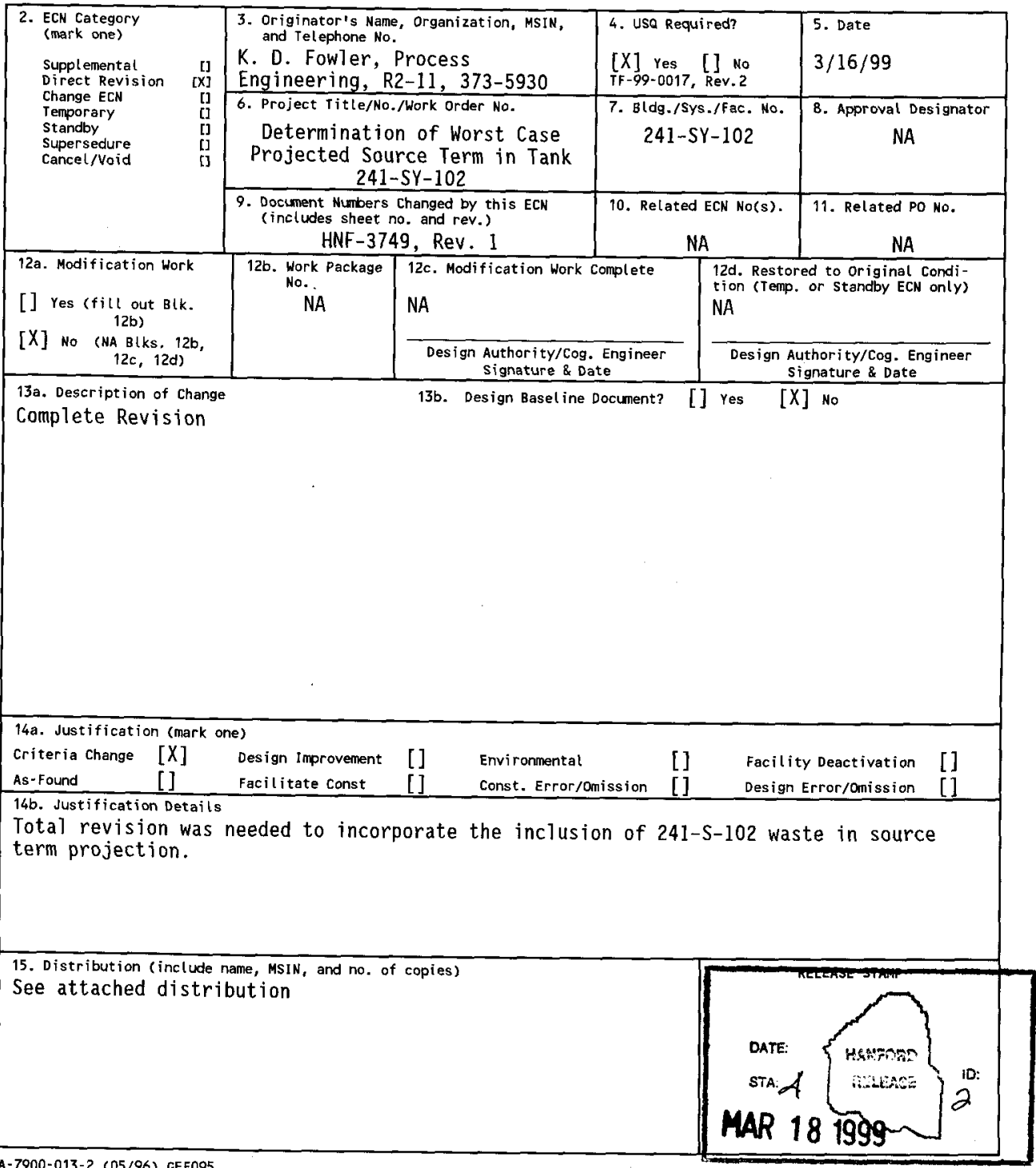




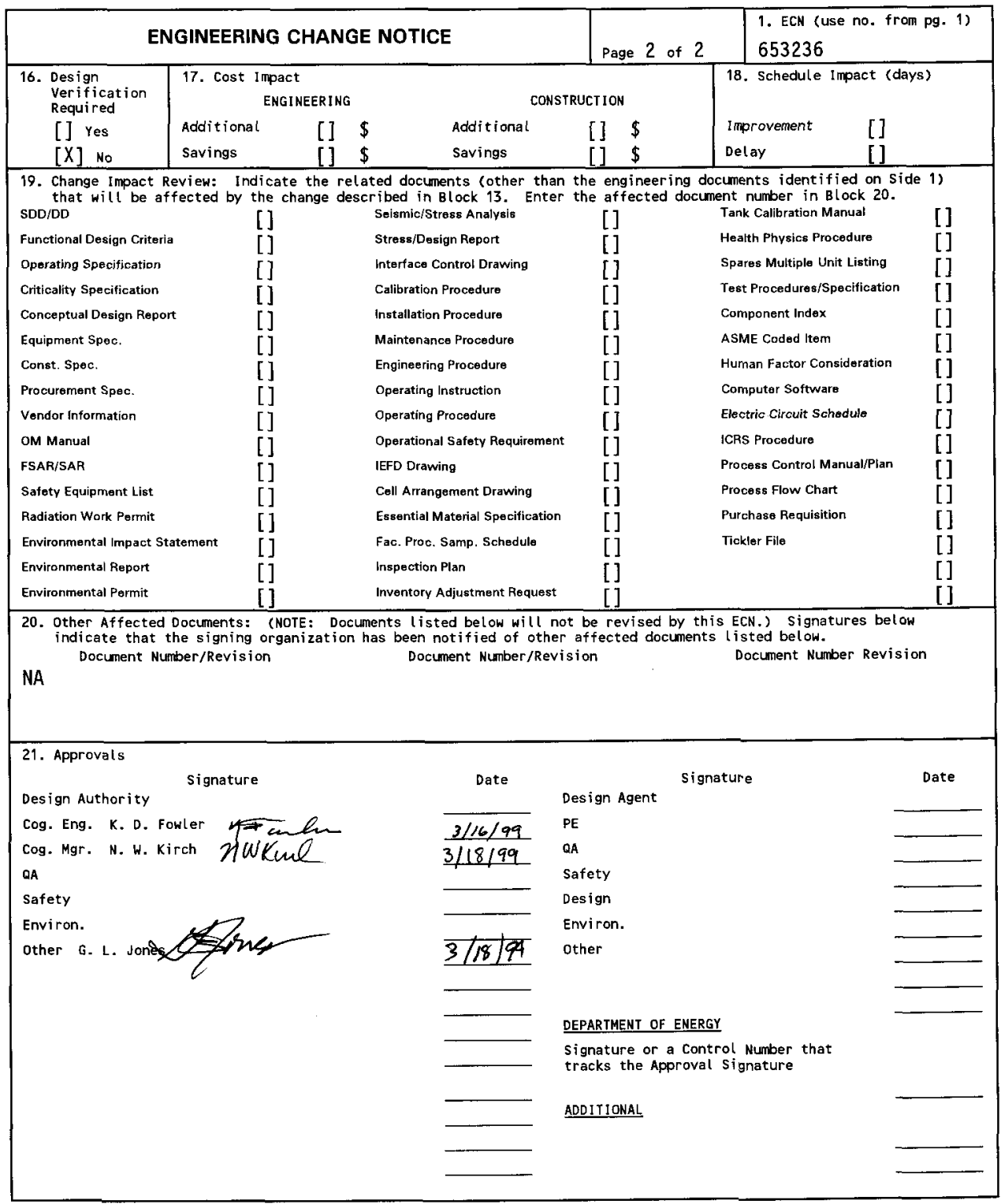


HNF-3749, Rev. 2

\section{Determination of Worst Case Projected Source Term in Tank 241-SY-102}

K. D. Fowler

Lockheed Martin Hanford, Corp., Richland, WA 99352

U.S. Department of Energy Contract DE-AC06-96RL13200

EDT/ECN: ECN-653236 UC: 2070

Org Code: 74B50 Charge Code: 101950

B\&R Code: EW 3120074 Total Pages: 7

Key Words: Cross-Site, 24I-SY-102, source term, W-058

Abstract: This document describes the methodology used to determine the worst case projected source term that could be in double-she 11 tank 241-SY-102 for the upcoming cross-site waste transfer.

TRADEMARK DISCLAIMER. Reference herein to any specific commercial product, process, or service by trade name, trademark, manufacturer, or otherwise, does not necessarily constitute or imply its endorsement, recomendation, or favoring by the United States Government or any agency thereof or its contractors or subcontractors.

Printed in the United States of America. To obtain copies of this document, contact: Document Control Services, P.0. Box 950, Mailstop H6-08, Richland WA 99352, Phone (509) 372-2420;

Fax (509) 376-4989.
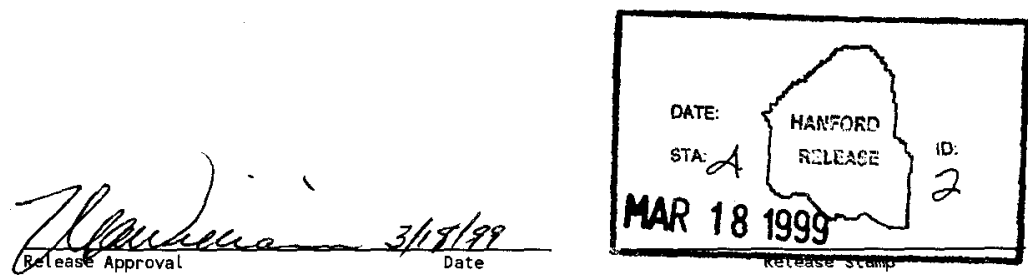

\section{Approved for Public Release}


(2) Title

Determination of Worst Case Projected Source Term in Tank 241-SY-102

CHANGE CONTROL RECORD

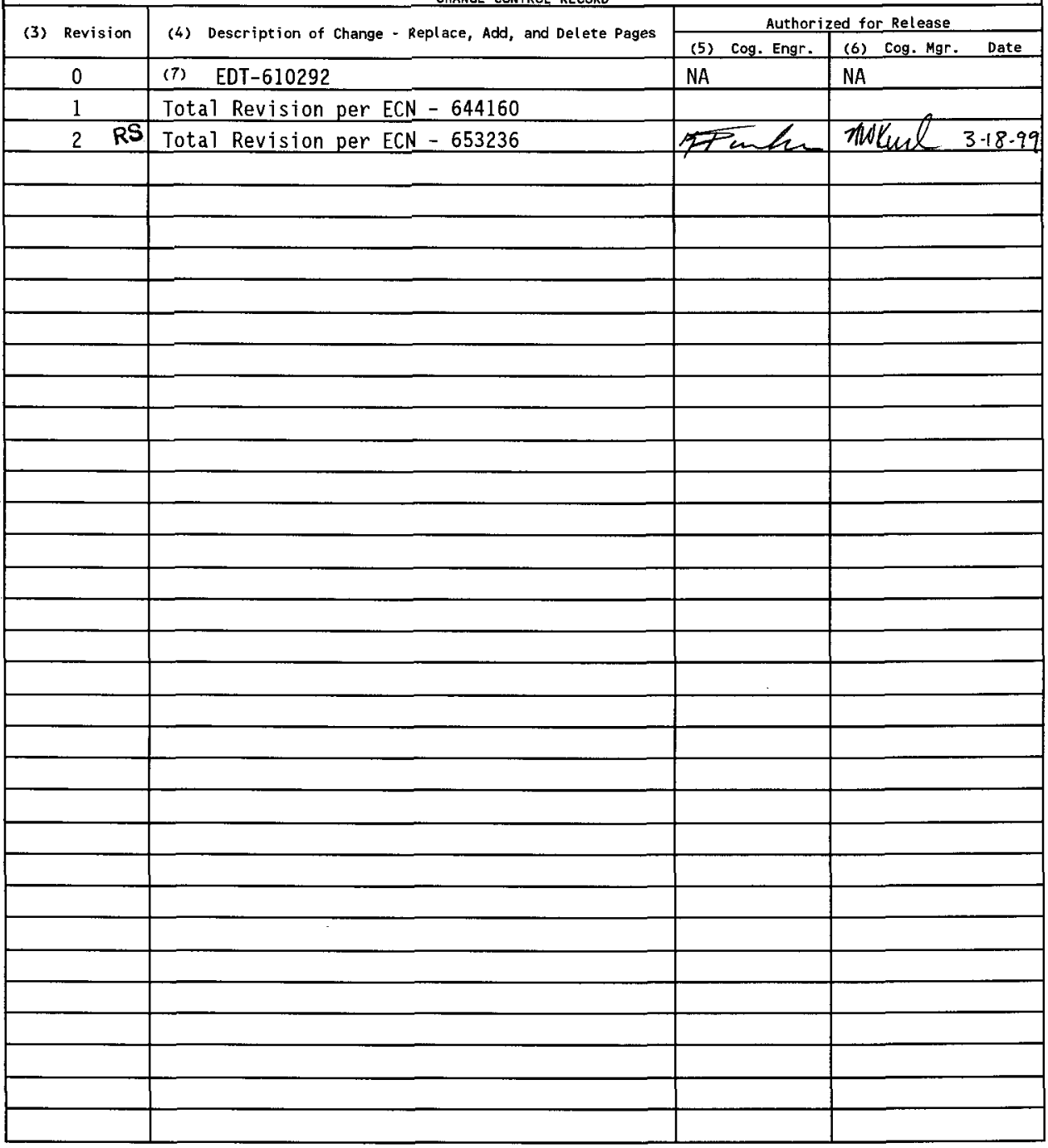


HNF-3749, Rev. 2

\section{Determination of Worst Case Projected Source Term in Tank 241-SY-102 Waste}

\subsection{Introduction}

This document describes the methodology used to determine the worst case projected source term that could be in double-shell tank 241-SY-102 for the cross-site waste transfer to tank 241-AP-107. The worst case projected source term given in Table 4-1 is the weighted average of the tank waste sources with the addition of sufficient waste from a defined worst source tank to fill the tank to 416 inches.

The projected source term in this document is not intended to represent what will be in tank 241-SY-102. Rather, it is a conservative bounding composition considering that the volume of waste that will be added from each current source is unknown.

\subsection{Enabling Assumptions}

In order to project the worst case source term for tank 241-SY-102 the following enabling assumptions were used.

- Tank 241-SY-102 would be filled to a maximum of 416 inches prior to the start of the crosssite transfer.

- The only sources of waste into tank 241-SY-102 since September 22, 1998 are tanks 241-S-102, 241-SX-104, 241-SX-106, 241-T-104, 241-T-110 and 1,200 gallons of 222-S Laboratory waste.

- The concentration of analytes of interest in water added to tank 241-SY-102 or to any of the source tanks is 0 .

- The concentration of analytes for which no analytical data or Best Basis inventory (Reference 1) value was available was assumed to be 0 .

- The concentration of undetected analytes was assumed to be 0 .

- The worst source tank is defined as a tank containing the highest concentration from among the source tanks for each analyte. Concentrations in 222-S Laboratory waste are not considered in defining the worst source tank because the volume of this waste is limited to a maximum of 1,200 gallons. 


\subsection{Tank Waste Material Balance}

To perform the worst case source term projection September 22, 1998 was chosen as the starting date because it is the date of the most recent waste sampling event in tank 241-SY-102. Data from these samples were used to determine the baseline composition of the tank. A material balance was done to account for the waste and water added to tank 241-SY-102 between the September 22, 1998 sampling and December 6, 1998.

December 6, 1998 was chosen arbitrarily as the cut off date for assessing current waste source contributions to tank 241-SY-102. The tank ENRAF waste level readings taken from the Surveillance Analysis Computer System (SACS) database were used to calculate the waste volumes using the conversion factor of 2754 gallons per inch.

Table 3-1 shows the volumes from the various sources used in the material balance. The "Check" value at the bottom of the table represents the difference between the volume of documented water and waste sources added to tank 241-SY-102 and the tank waste volume on the cut off date. The value represents approximately 0.02 inch and is not significant for the purpose of this projection.

Table 3-1

\begin{tabular}{|c|c|c|}
\hline Volumes & Date & Gallons \\
\hline 241-SY-102 Waste Level from ENRAF Reading & $12 / 06 / 98$ & 965194 \\
\hline 241-SY-102 Waste Level from ENRAF Reading & $09 / 22 / 98$ & 844679 \\
\hline \multicolumn{3}{|l|}{ Sources } \\
\hline 210-SX-104 (Process Engineering Pu Inventory Database) & & 23717 \\
\hline 241-SX-106 (Process Engineering Pu Inventory Database) & & 12167 \\
\hline 241-T-104 (Process Engineering Pu Inventory Database) & & 12099 \\
\hline 241-T-110 (Process Engineering Pu Inventory Database) & & 10399 \\
\hline Raw Water (Operations Database) & & 42093 \\
\hline Rain Water (Process Engineering Pu Inventory Database) & & 20098 \\
\hline TOTAL (9/22/98 Waste Level + sum of sources) & & 965252 \\
\hline Check (TOTAL - 12/6/98 Waste Level) & & 58 \\
\hline
\end{tabular}

\subsection{Source Term Determination}

In order to reflect that a partial cross-site transfer has occurred, the difference between the March 10, 1999 and the March 14, 1999 ENRAF waste level readings from SACS (406 and 355 inches, respectively) was determined. This volume was used to calculate the percentage of waste removed from the tank and thus, the percentage decrease in each of the source wastes.

The worst case projected source term in tank 241-SY-102 was determined with the assumption that the tank will be filled to 416 inches of waste. For each analyte, the products of the baseline concentration in tank 241-SY-102, one of the source wastes or the defined worst source tank with the fraction of that waste in an assumed full tank 241-SY-102 were added together. These weighted averages represent the worst case projected source term. These values along with the concentrations used to calculate them are given in Table 4-1. 
In most cases, data from the September 22, 1998 tank waste samples (Reference 2) were used as the baseline composition of tank 241-SY-102. The concentration of undetected analytes was assumed to be 0 . For analytes where no data was available, the Best Basis inventory was used to calculate the concentration. If no Best Basis inventory value was available, the concentration was assumed to be 0 . The baseline waste volume for tank 241-SY-102 was calculated from the September 22, 1998 ENRAF waste level reading taken from the SACS database.

For tanks 241-S-102, 241-SX-104, 241-SX-106, 241-T-104, and 241-T-110, analyte concentrations were obtained from the Tank Characterization Database (TCD). For the purpose of this projection, the concentrations of undetected analytes are assumed to be 0 . Where no data were available, the Best Basis tank inventory was used to calculate the concentration. Where no Best Basis inventory value was available, the concentration was assumed to be 0 .

For 222-S Laboratory waste, analyte concentrations were obtained from the sample data included as an attachment to Reference 1. For the purpose of this projection, the concentrations of unreported analytes and undetected analytes are assumed to be 0 . 
HNF-3749, Rev. 2

Table 4-1

\begin{tabular}{|c|c|c|c|c|c|c|c|c|c|c|c|c|}
\hline & UNIT & SY-102 & S-102 & sX-104 & SX-106 & T-104 & $\mathrm{T}-110$ & 222-S & Water & $\begin{array}{l}\text { Worst } \\
\text { Case Tank }\end{array}$ & $\begin{array}{l}\text { Wh. } \\
\text { Average }\end{array}$ & $\begin{array}{l}\text { Bounding } \\
\text { Case } \\
\text { (Bql) }\end{array}$ \\
\hline Fraction & & 6.3E-01 & $0.0 E+00$ & $1.8 \mathrm{E}-02$ & 9.1E-03 & $9.0 \mathrm{E}-03$ & $7.7 \mathrm{E}-03$ & $8.9 \mathrm{E}-04$ & $4.6 \mathrm{E}-02$ & $2.8 \mathrm{E}-01$ & & \\
\hline Conversion Vol (l) & I & $1.1 \mathrm{E}+06$ & & & $2.0 \mathrm{E}+06$ & $1.7 \mathrm{E}+06$ & \begin{tabular}{|l|}
$1.4 \mathrm{E}+06$ \\
\end{tabular} & $7.6 E+03$ & & & & \\
\hline Max SpG & & 1.16 & 1.55 & 1.49 & 1.50 & 1.30 & \begin{tabular}{|l|}
1.11 \\
\end{tabular} & 1.08 & 1.0 & 1.55 & 1.27 & \\
\hline Co-60 & $\mathrm{Ci} / 1$ & $2.6 \mathrm{E}-06$ & \begin{tabular}{|l|}
$0.0 E+00$ \\
\end{tabular} & $0.0 \mathrm{E}+00$ & \begin{tabular}{|l|}
$0.0 \mathrm{E}+00$ \\
\end{tabular} & $0.0 \mathrm{E}+00$ & $0.0 \mathrm{E}+00$ & $2.6 \mathrm{E}-07$ & $0.0 \mathrm{E}+00$ & $0.0 \mathrm{E}+00$ & $1.7 \mathrm{E}-06$ & $6.1 E+04$ \\
\hline Sr-90 (89/90) & $\mathrm{Ci} / \mathrm{I}$ & $5.0 \mathrm{E}-06$ & \begin{tabular}{|c|}
$3.0 \mathrm{E}-04$ \\
\end{tabular} & $6.5 \mathrm{E}-05$ & \begin{tabular}{|l|}
$4.2 \mathrm{E}-04$ \\
\end{tabular} & 3.4E-03 & 5.6E-07 & 6.7E-04 & $0.0 \mathrm{E}+00$ & 3.4E-03 & $9.9 \mathrm{E}-04$ & $3.7 E+07$ \\
\hline Y-90 (from Sr) & $\mathrm{Ci} / \mathrm{I}$ & $5.0 \mathrm{E}-06$ & 3.0E-04 & 6.5E-05 & $4.2 \mathrm{E}-04$ & $3.4 \mathrm{E}-03$ & $5.6 \mathrm{E}-07$ & $6.7 \mathrm{E}-04$ & $0.0 \mathrm{E}+00$ & $3.4 \mathrm{E}-03$ & 9.9E-04 & $3.7 \mathrm{E}+07$ \\
\hline Cs-137 & $\mathrm{Ci} / \mathrm{h}$ & 3.7E-02 & $8.0 \mathrm{E}-02$ & $2.3 \mathrm{E}-01$ & $4.1 \mathrm{E}-01$ & 8.1E-05 & $3.6 \mathrm{E}-06$ & 7.4E-04 & $0.0 \mathrm{E}+00$ & 4.1E-01 & $1.5 \mathrm{E}-01$ & $5.4 E+09$ \\
\hline Eu-154 & $\mathrm{Ci} / \mathrm{I}$ & $2.0 \mathrm{E}-07$ & & $9.2 \mathrm{E}-05$ & $6.5 \mathrm{E}-04$ & $3.5 \mathrm{E}-07$ & $1.3 \mathrm{E}-10$ & & $0.0 \mathrm{E}+00$ & 6.5E-04 & $1.9 \mathrm{E}-04$ & $7.0 \mathrm{E}+06$ \\
\hline $\mathrm{Np}-237$ & $\mathrm{Ci}$ & & & 8.7E-07 & $9.4 \mathrm{E}-07$ & $9.0 \mathrm{E}-09$ & $5.0 \mathrm{E}-10$ & & $0.0 \mathrm{E}+00$ & $9.4 \mathrm{E}-07$ & $7.8 \mathrm{E}-07$ & $2.9 \mathrm{E}+04$ \\
\hline Pu-238 & $\mathrm{Ci} / 1$ & $8.4 \mathrm{E}-12$ & & $2.6 \mathrm{E}-06$ & $3.2 \mathrm{E}-06$ & $9.0 \mathrm{E}-08$ & 2.1E-09 & & $0.0 \mathrm{E}+00$ & $3.2 \mathrm{E}-06$ & 9.6E-07 & $3.6 \mathrm{E}+04$ \\
\hline Pu-239 (239/40) & $\mathrm{Ci} / \mathrm{I}$ & $3.1 \mathrm{E}-07$ & $3.4 \mathrm{E}-08$ & $3.8 \mathrm{E}-08$ & 7.4E-08 & 9.4E-06 & 1.7E-07 & $1.6 \mathrm{E}-06$ & $0.0 \mathrm{E}+00$ & $4 E-06$ & $2.9 \mathrm{E}-06$ & $1.1 \mathrm{E}+05$ \\
\hline Pu-241 & $\mathrm{Ci} / \mathrm{l}$ & $7.2 \mathrm{E}-10$ & & $1.6 \mathrm{E}-04$ & $2.1 \mathrm{E}-04$ & $5.1 \mathrm{E}-05$ & $2.8 \mathrm{E}-05$ & & $0.0 \mathrm{E}+00$ & $2.1 E-04$ & 6.6E-05 & $2.4 \mathrm{E}+06$ \\
\hline Am-241 & $\mathrm{Ci} / \mathrm{I}$ & $2.6 \mathrm{E}-07$ & $2.8 \mathrm{E}-07$ & $0.0 \mathrm{E}+00$ & $0.0 \mathrm{E}+00$ & $0.0 \mathrm{E}+00$ & $0.0 \mathrm{E}+00$ & $3.6 \mathrm{E}-06$ & $0.0 \mathrm{E}+00$ & $2.8 \mathrm{E}-07$ & $2.5 \mathrm{E}-07$ & $9.1 \mathrm{E}+03$ \\
\hline \multirow[t]{4}{*}{$\mathrm{Cm}-244$} & $\mathrm{Ci}$ & $5.0 \mathrm{E}-11$ & & $1.3 \mathrm{E}-07$ & $3.1 \mathrm{E}-07$ & $3.2 E-08$ & 8.1E-09 & & $0.0 E+00$ & 3.1E-07 & $9.2 \mathrm{E}-08$ & $3.4 \mathrm{E}+03$ \\
\hline & & & & & & & & & & & Sum & $5.5 \mathrm{E}+09$ \\
\hline & & & & & & & & & & & & \\
\hline & & SY-102 & S-102 & SX-104 & SX-106 & $T-104$ & $T-110$ & $222-s$ & Water & $\begin{array}{l}\text { Worst Case } \\
\text { Tank }\end{array}$ & $\begin{array}{l}\text { Wt. } \\
\text { Average }\end{array}$ & $\begin{array}{l}\text { Bounding } \\
\text { Case } \\
(\mathrm{g} / \mathrm{l})\end{array}$ \\
\hline Ammonia (NH3) & $g /$ & $1.2 \mathrm{E}-01$ & $3.5 \mathrm{E}-02$ & $7.9 \mathrm{E}-01$ & $6.1 \mathrm{E}-02$ & $1.2 \mathrm{E}+00$ & $8.5 \mathrm{E}-05$ & 6.3E-03 & $0.0 \mathrm{E}+00$ & $1.2 \mathrm{E}+00$ & 4.3E-01 & 4.3E-01 \\
\hline Antimony (Sb) & $g / 1$ & $0.0 \mathrm{E}+00$ & $0.0 \mathrm{E}+00$ & $0.0 \mathrm{E}+00$ & $0.0 \mathrm{E}+00$ & $0.0 \mathrm{E}+00$ & $0.0 \mathrm{E}+00$ & $2.9 \mathrm{E}-03$ & $0.0 \mathrm{E}+00$ & $0.0 E+00$ & $2.6 \mathrm{E}-06$ & 2.6E-06 \\
\hline Arsenic (As) & $g / /$ & $0.0 \mathrm{E}+00$ & $0.0 \mathrm{E}+00$ & & & & & $0.0 \mathrm{E}+00$ & $0.0 \mathrm{E}+00$ & $0.0 \mathrm{E}+00$ & $0.0 \mathrm{E}+00$ & $0.0 \mathrm{E}+00$ \\
\hline Barium (Ba) & $g / 1$ & $0.0 \mathrm{E}+00$ & $0.0 E+00$ & $0.0 \mathrm{E}+00$ & $0.0 \mathrm{E}+00$ & $0.0 \mathrm{E}+00$ & $0.0 \mathrm{E}+00$ & 4.8E-04 & $0.0 \mathrm{E}+00$ & $0.0 \mathrm{E}+00$ & 4.3E-07 & 4.3E-07 \\
\hline Beryllium (Be) & $g / /$ & $0.0 \mathrm{E}+00$ & $0.0 \mathrm{E}+00$ & $0.0 \mathrm{E}+00$ & $0.0 E+00$ & $0.0 \mathrm{E}+00$ & $0.0 \mathrm{E}+00$ & $2.9 \mathrm{E}-05$ & $0.0 \mathrm{E}+00$ & $0.0 \mathrm{E}+00$ & $2.6 E-08$ & $2.6 \mathrm{E}-08$ \\
\hline Cadmium (Cd) & $g / 1$ & $0.0 \mathrm{E}+00$ & $0.0 \mathrm{E}+00$ & $0.0 \mathrm{E}+00$ & $0.0 \mathrm{E}+00$ & $0.0 E+00$ & $0.0 \mathrm{E}+00$ & $7.0 \mathrm{E}-04$ & $0.0 E+00$ & $0.0 \mathrm{E}+00$ & $6.3 \mathrm{E}-07$ & 6.3E-07 \\
\hline Calcium (Ca) & $g / l$ & $0.0 \mathrm{E}+00$ & $0.0 \mathrm{E}+00$ & $0.0 \mathrm{E}+00$ & $0.0 \mathrm{E}+00$ & $0.0 \mathrm{E}+00$ & $0.0 \mathrm{E}+00$ & $3.4 \mathrm{E}-02$ & $0.0 \varepsilon+00$ & $0.0 \mathrm{E}+00$ & 3.0E-05 & $3.0 \mathrm{E}-05$ \\
\hline Cerium (Ce) & $g / 1$ & $0.0 \mathrm{E}+00$ & $0.0 E+00$ & $0.0 \mathrm{E}+00$ & $0.0 E+00$ & & $0.0 \mathrm{E}+00$ & $0.0 \mathrm{E}+00$ & $0.0 \mathrm{E}+00$ & $0.0 \mathrm{E}+00$ & $0.0 \mathrm{E}+00$ & $0.0 \mathrm{E}+00$ \\
\hline Chromium $(\mathrm{Cr}+3)$ & $g / /$ & $6.7 E-01$ & $4.7 \mathrm{E}-01$ & $1.1 \mathrm{E}+00$ & $1.4 \mathrm{E}-01$ & $3.2 E-01$ & 3.5E-02 & $6.7 \mathrm{E}-03$ & $0.0 \mathrm{E}+00$ & $1.1 \mathrm{E}+00$ & $7.4 \mathrm{E}-01$ & 7.4E-01 \\
\hline Cobalt (Co) & $g^{/ /}$ & $0.0 \mathrm{E}+00$ & $0.0 \mathrm{E}+00$ & $0.0 \mathrm{E}+00$ & $0.0 \mathrm{E}+00$ & $0.0 \mathrm{E}+00$ & $0.0 \mathrm{E}+00$ & $0.0 \mathrm{E}+00$ & $0.0 E+00$ & $0.0 \mathrm{E}+00$ & $0.0 \mathrm{E}+00$ & $0.0 E+00$ \\
\hline Cyanide (CN) & $g / 1$ & & & & & $7.2 \mathrm{E}-04$ & & $2.6 \mathrm{E}-04$ & $0.0 \mathrm{E}+00$ & $7.2 \mathrm{E}-04$ & $2.1 \mathrm{E}-04$ & 2.1E-04 \\
\hline Dysprosium (Dy) & g/ $/$ & & & & & & & & $0.0 \mathrm{E}+00$ & $0.0 E+00$ & $0.0 \mathrm{E}+00$ & $0.0 \mathrm{E}+00$ \\
\hline Lanthanum (La) & $g / /$ & $0.0 \mathrm{E}+00$ & $0.0 \mathrm{E}+00$ & $0.0 \mathrm{E}+00$ & $0.0 \mathrm{E}+00$ & 1.7E-05 & $0.0 \mathrm{E}+00$ & $1.2 \mathrm{E}-03$ & $0.0 \mathrm{E}+00$ & $1.7 \mathrm{E}-05$ & $5.8 \mathrm{E}-06$ & $5.8 \mathrm{E}-06$ \\
\hline Mercury $(\mathrm{Hg})$ & $g / 1$ & $0.0 \mathrm{E}+00$ & & $0.0 E+\infty 0$ & $0.0 \mathrm{E}+00$ & 1.6E-04 & $0.0 \mathrm{E}+00$ & $1.5 \mathrm{E}-05$ & $0.0 \mathrm{E}+00$ & $1.6 \mathrm{E}-04$ & 4.7E-05 & $4.7 \mathrm{E}-05$ \\
\hline Neodymium Nd) & $g / 1$ & $0.0 \mathrm{E}+00$ & $0.0 \mathrm{E}+00$ & $0.0 E+00$ & $0.0 \mathrm{E}+00$ & & $0.0 \mathrm{E}+00$ & $0.0 E+00$ & $0.0 \mathrm{E}+00$ & $0.0 \mathrm{E}+00$ & $0.0 \mathrm{E}+00$ & $0.0 \mathrm{E}+00$ \\
\hline \begin{tabular}{|l|} 
Oxalate (C2O4) \\
\end{tabular} & $\mathrm{g} / \mathrm{l}$ & $0.0 \mathrm{E}+00$ & $5.8 \mathrm{E}-01$ & $1.1 \mathrm{E}+00$ & $1.2 \mathrm{E}+00$ & $1.4 \mathrm{E}-05$ & $0.0 \mathrm{E}+00$ & $0.0 \mathrm{E}+00$ & $0.0 E+00$ & $1.2 \mathrm{E}+00$ & 3.5E-01 & $3.5 \mathrm{E}-01$ \\
\hline Selenium (Se) & $g / l$ & $0.0 \mathrm{E}+00$ & $0.0 E+00$ & $0.0 \mathrm{E}+00$ & $0.0 \mathrm{E}+00$ & $0.0 E+00$ & $0.0 E+00$ & $0.0 \mathrm{E}+00$ & $0.0 \mathrm{E}+00$ & $0.0 \mathrm{E}+00$ & $0.0 E+00$ & $0.0 \mathrm{E}+00$ \\
\hline Sodium Hydroxide & $g / l$ & $1.1 \mathrm{E}+01$ & $8.9 \mathrm{E}+01$ & $9.3 E+01$ & $9.0 \mathrm{E}+01$ & $1.5 \mathrm{E}+02$ & $7.9 \mathrm{E}+01$ & $4.3 E+01$ & $0.0 \mathrm{E}+00$ & $1.5 \mathrm{E}+02$ & $5.3 \mathrm{E}+01$ & $5.3 E+01$ \\
\hline Sodium - NaOH & $g / 1$ & $4.0 E+01$ & $1.4 \mathrm{E}+02$ & $2.0 \mathrm{E}+02$ & $2.2 \mathrm{E}+02$ & $0.0 E+00$ & $0.0 \mathrm{E}+00$ & $0.0 \mathrm{E}+00$ & $0.0 \mathrm{E}+00$ & $2.2 \mathrm{E}+02$ & $9.2 \mathrm{E}+01$ & $2 \mathrm{E}+01$ \\
\hline Tellurium (Te) & $g / 1$ & & & & & & & & $0.0 E+00$ & $0 E+00$ & $0.0 \mathrm{E}+00$ & $0.0 \mathrm{E}+00$ \\
\hline Thallium (T1) & $g / 1$ & $0.0 \mathrm{E}+00$ & $0.0 \mathrm{E}+00$ & $0.0 \mathrm{E}+00$ & $0.0 \mathrm{E}+00$ & & $0.0 \mathrm{E}+00$ & $0.0 \mathrm{E}+00$ & $0.0 \mathrm{E}+00$ & $.0 \mathrm{E}+00$ & $0.0 \mathrm{E}+00$ & $0.0 \mathrm{E}+00$ \\
\hline TOC-Oxalate & $9 / 1$ & $0.0 E+00$ & $2.4 \mathrm{E}+00$ & $1.2 \mathrm{E}+00$ & $3.4 \mathrm{E}+00$ & 4.7E-01 & $4.5 \mathrm{E}-02$ & $4.0 \mathrm{E}-01$ & $0.0 \mathrm{E}+00$ & $3.4 \mathrm{E}+00$ & $1.0 \mathrm{E}+00$ & $1.0 \mathrm{E}+00$ \\
\hline Uranium (U) & $g / 1$ & $1.8 \mathrm{E}-02$ & $0.0 \mathrm{E}+00$ & $3.0 \mathrm{E}-01$ & \begin{tabular}{|c|}
$3.0 \mathrm{E}-01$ \\
\end{tabular} & $1.2 \mathrm{E}+00$ & $5.0 \mathrm{E}-02$ & $1.2 \mathrm{E}-02$ & $0.0 E+00$ & $2 E+00$ & $3.6 \mathrm{E}-01$ & $3.6 \mathrm{E}-01$ \\
\hline Vanadium $(\mathrm{V})$ & $g h$ & $0.0 \mathrm{E}+00$ & $0.0 \mathrm{E}+00$ & $0.0 \mathrm{E}+00$ & $0.0 \mathrm{E}+00$ & & $0.0 \mathrm{E}+00$ & $0.0 \mathrm{E}+00$ & $0.0 E+00$ & $0.0 \mathrm{E}+00$ & $0.0 \mathrm{E}+00$ & $0.0 \mathrm{E}+00$ \\
\hline & & & & & & & & & & & & \\
\hline Hydroxide $(\mathrm{OH})$ & $g /$ & $4.8 E+00$ & $3.8 \mathrm{E}+01$ & $4.0 \mathrm{E}+01$ & $3.8 \mathrm{E}+01$ & $6.3 E+01$ & $3.4 \mathrm{E}+01$ & $1.8 \mathrm{E}+01$ & & $6.3 \mathrm{E}+01$ & $2.3 \mathrm{E}+01$ & $2.3 E+01$ \\
\hline $\mathrm{Na}$ as $\mathrm{NaOH}$ (from $\mathrm{OH}$ ) & $g / 1$ & $6.5 \mathrm{E}+00$ & $5.1 \mathrm{E}+01$ & $5.4 E+01$ & $5.2 \mathrm{E}+01$ & $8.5 \mathrm{E}+01$ & $4.6 \mathrm{E}+01$ & $2.5 E+01$ & & $8.5 \mathrm{E}+01$ & $3.1 \mathrm{E}+01$ & $3.1 \mathrm{E}+01$ \\
\hline
\end{tabular}


HNF-3749, Rev. 2

\subsection{References}

1. Agnew, S. F., 1997, Hanford Tank Chemical and Radionuclide Inventories: HDW Model Rev. 4, "LA-UR-96-3860, dated January 1997.

2. Fuller, R. K, 1998, “Compatibility Interim Results for Tank 241-SY-102 Grab Samples," Letter WMH-9859910 to K. M. Hall, LMHC, dated November 18, 1998.

3. McDowell, A. K., "Waste Transfer Documentation for Shipment 99-01 of 219-S Tank System Laboratory Waste," Letter WMH-9950456 to C. B. Bryan, LMHC. 


\section{DISTRIBUTION SHEET}

\begin{tabular}{|c|c|c|c|c|c|}
\hline \multirow{2}{*}{$\begin{array}{l}\text { To } \\
\text { Distribution }\end{array}$} & \multirow{2}{*}{\multicolumn{3}{|c|}{$\begin{array}{l}\text { From } \\
\text { Process Engineering }\end{array}$}} & \multicolumn{2}{|l|}{ Page 1 of 1} \\
\hline & & & & \multicolumn{2}{|c|}{ Date $3 / 16 / 99$} \\
\hline \multicolumn{4}{|l|}{ Project Title/Work Order } & \multicolumn{2}{|l|}{ EDT No. NA } \\
\hline \multicolumn{4}{|c|}{$\begin{array}{l}\text { HNF-3749, Rev. } 2 \text { "Determinaiton of Worst Case Projected Source } \\
\text { Term in Tank 241-SY-102 }\end{array}$} & \multicolumn{2}{|c|}{ ECN No. 653236} \\
\hline Name & MSIN & $\begin{array}{c}\text { Text } \\
\text { With All } \\
\text { Attach. }\end{array}$ & Text Only & $\begin{array}{l}\text { Attach./ } \\
\text { Appendix } \\
\text { Only }\end{array}$ & $\begin{array}{l}\text { EDT/ECN } \\
\text { Only }\end{array}$ \\
\hline
\end{tabular}

\section{ONSITE}

DE\&S Hanford, Inc.

G. W. Gault R1-44X

Lockheed Mart in Hanford, Corp.

C. DeFigh-Price R2-12 X

K. D. Fowler R2-11 $X$

N. W. Kirch R2-11 $X$

D. A. Reynolds R2-11 $X$

T.C.S.R.C R1-10

Lockneed Mart in Services. Inc.

Central Files Bl-07 $X$

MACTEC

G. L. Jones RI-44X 